\title{
Comparison of Monte Carlo Techniques for Obtaining System-Reliability Confidence Limits
}

\author{
Albert H. Moore \\ Air Force Institute of Technology, \\ Wright-Patterson AFB \\ H. Leon Harter \\ Wright State University, Dayton \\ Robert C. Snead \\ Tracor, Inc., Austin
}

Key Words-Monte Carlo, s-Confidence limit, Gamma distribution, Weibull distribution, Logistic distribution.

\author{
Reader Aids- \\ Purpose: Widen state of art \\ Special math needed for explanations: Probability and statistics \\ Special math needed for results: Statistics \\ Results useful to: Reliability theoreticians
}

Summary \& Conclusion-Digital computer techniques are developed using a) asymptotic distributions of maximum likelihood estimators, and b) a Monte Carlo technique, to obtain approximate system reliability $s$-confidence limits from component test data. 2-Parameter Weibull, gamma, and logistic distributions are used to model the component failures. The components can be arranged in any system configuration: series, parallel, bridge, etc., as long as one can write the equation for system reliability in terms of component reliability. Hypothetical networks of 3 5 , and 25 components are analyzed as examples. Univariate and bivariate asymptotic techniques are compared with a double Monte Carlo method. The bivariate asymptotic technique is shown to be fast and accurate. It can guide decisions during the research and development cycle prior to complete system testing and can be used to supplement system failure data.

\section{INTRODUCTION}

The problem of obtaining system s-confidence limits from subsystem test data has been studied extensively. Springer \& Thompson [24-26] developed Bayesian limits for serially connected subsystems whose failure models follow the binomial or exponential distributions by using Mellin transforms. Lannon [14] and Lutton [18] used a technique based on the asymptotic $s$-normality of maximum likelihood (ML) estimators. Mann \& Grubbs [20] discussed approximately optimum $s$-confidence limits for series or parallel systems with binomial or exponentially distributed failure times. For binomial failure data they also discussed an asymptotic technique for logically complex $s$-coherent systems. Berkbigler \& Byers [2] compared "two of the most widely used techniques for computing lower limits for the reliability of series subsystems, the exact Bayesian limits of Springer \& Thompson [24] and the fiducial limits using Monte Carlo simulation [15]'”. Locks $[16,17]$ developed Monte Carlo Bayesian programs for establishing interval estimates for reliability and the MTBF for a large and complex system of any modular configura- tion. O'Neil [22] examined some methods of estimating lower $s$-confidence limits on system reliability for serial systems based on subsystem test data. Gatliffe [7] used a log-gamma method to find system $s$-confidence limits. His analysis goes beyond the original log-gamma method which was designed solely for serial systems. Levy \& Moore [15] designed a digital computer process to obtain system reliability $s$-confidence limits for a system composed of different elements whose failures followed different probability distributions. The distributions of the estimators of the parameters were assumed to be known for the cases considered. Moore [21] discussed a general Monte Carlo technique to obtain system reliability $s$-confidence limits when the distribution of the estimators is unknown. The double Monte Carlo method is related to the "bootstrap" method of B. Efron [6]. Another way to approximate the distribution of the estimators is to use the asymptotic distribution of the estimators. Harter \& Moore [8-13] derived ML estimators and their covariance matrices for complete and censored samples from the $s$-normal, Weibull, gamma, logistic, lognormal, and first asymptotic distribution of extreme values. Bilikam \& Moore [3, 4] derived ML estimators and their covariance matrices for multiple independent grouped censored samples from the Weibull distribution with failure times known and unknown.

This paper compares three Monte Carlo techniques to obtain system reliability $s$-confidence limits from component test data. Two of the techniques use the asymptotic $s$-normality property of ML estimators while the other uses a Monte Carlo technique to obtain the approximate distributions of the estimators. In addition, for systems with three components, the true $s$-confidence levels are compared for the two asymptotic methods with the desired $s$-confidence levels.

\section{METHODOLOGY}

The two assumptions common to the three methods are:

1) The underlying life distribution family is known for each component.

2) Components have been life tested. All unknown parameters of the life distributions have been estimated from the data. For the gamma and Weibull distributions it is assumed that the location parameter is known.

\section{Notation}

DOU Double Monte Carlo Method

BIV Bivariate Asymptotic Method

UNI Univariate Asymptotic Method

$t_{m} \quad$ given mission time for system

$n \quad$ sample size for component test data 
$(\hat{\alpha}, \hat{\beta})$ maximum likelihood (ML) estimates of the parameters $(\alpha, \beta)$ obtained from component test data

$\hat{R} \quad$ ML estimate of reliability of component

$V$ asymptotic covariance matrix of bivariate distribution of $(\hat{\alpha}, \hat{\beta})$

$V_{R}, \operatorname{Var}(\hat{\alpha}), \operatorname{Var}(\hat{\beta})$ asymptotic variance of $\hat{R}, \hat{\alpha}$, and $\hat{\beta}$

$Z_{i} \quad$ simulated realizations of $s$-independent standard normal deviates

$\left(\alpha_{i}, \beta_{i}\right)$ simulated realizations of parameter vector $(\alpha, \beta)$

$(\theta, B)$ scale and shape parameters of Weibull distribution

$(\gamma, \alpha)$ scale and shape parameters of gamma distribution

$(\mu, \sigma)$ location and scale parameters of logistic distribution

Other, standard notation is given in "Information for Readers \& Authors" at the rear of each issue.

Double Monte Carlo Method [21]. Samples are generated whose failures have the same distribution and parameters as the estimated ones and the same number of observations as the original test data. The parameters are estimated from the simulated sample by the same method as used on the original sample. The simulated values of the parameters and the mission time are substituted in the life distribution to obtain a simulated reliability for each component.

Bivariate Asymptotic Method. The simulated values of the parameters of the failure model are generated by (1);

$\left(\begin{array}{c}\alpha_{i} \\ \beta_{i}\end{array}\right)=V^{1 / 2}\left(\begin{array}{c}z_{i_{1}} \\ z_{i 2}\end{array}\right)+\left(\begin{array}{c}\hat{\alpha} \\ \hat{\beta}\end{array}\right)$

The simulated values of the parameters and the mission time are substituted in the life distribution to obtain a simulated reliability for each component.

Univariate Asymptotic Method. The variance of a 2-parameter function of ML estimators is asymptotically-

$$
\begin{aligned}
& \operatorname{Var}\{R(\hat{\alpha}, \hat{\beta})\}=(\partial R / \partial \hat{\alpha})^{2} \operatorname{Var}\{\hat{\alpha}\}+(\partial R / \partial \hat{\beta})^{2} \operatorname{Var}\{\hat{\beta}\} \\
& +2(\partial R / \partial \hat{\alpha})(\partial R / \partial \hat{\beta}) \operatorname{Cov}\{\hat{\alpha}, \hat{\beta}\}
\end{aligned}
$$

For a simulated value of the reliability we obtain an equation similar to (1)

$R_{i}=\left(V_{\hat{R}}\right)^{1 / 2} Z_{i}+\hat{R}$

By inserting the system reliability equation in terms of component reliabilities and using the univariate asymptotic distribution of each $R_{i}$ as the actual distribution, one could use the Monte Carlo method called "sample" [27] to obtain the distribution of system reliability and hence system $s$-confidence intervals corresponding to those obtained by the univariate asymptotic method.

\section{ILLUSTRATIVE SYSTEMS}

Hypothetical networks of 3, 5, and 25 components are analyzed as examples with an assumed mission time of 100 hours. Five network configurations were considered as follows: 3 components in series; 1 in series with 2 in parallel; 3 components in parallel; 5-component network (see Fig. 1); and 25-component network (see Fig. 2). The life distributions are-
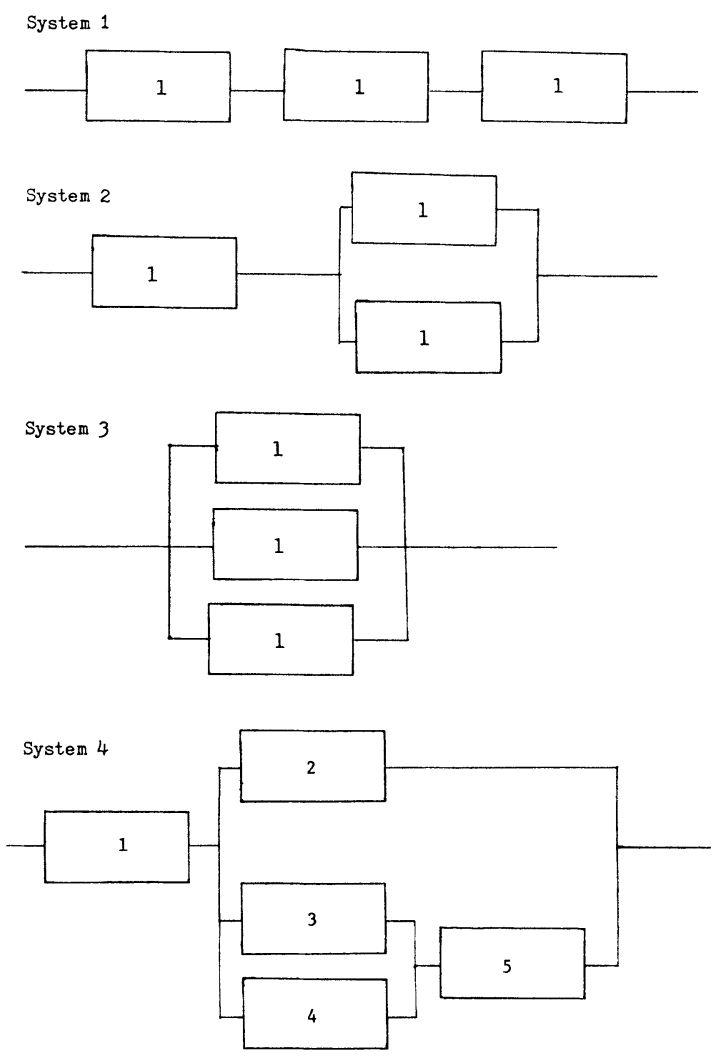

Fig. 1. Systems 1, 2, 3, and 4 .
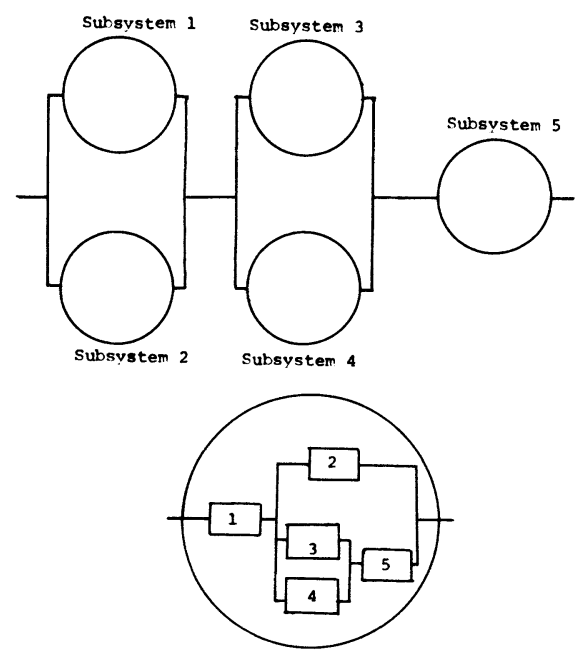

Each subsvstem configuration

Fig. 2. 25 Component Network. 
Weibull: $R\left(t_{m}\right)=\exp \left[-\left(t_{m} / \theta\right)^{B}\right]$; location parameter $=0$

Logistic: $R\left(t_{m}\right)=1-\left[1+\exp \left\{-\pi\left(t_{m}-\mu\right) /(\sqrt{3 \sigma})\right\}\right]^{-1}$

Gamma: $R\left(t_{m}\right)=1-\int_{0}^{t_{m} / \gamma} b(u) d u$.

$b(u) \equiv\left[u^{\alpha-1} \exp (-u)\right] / \Gamma(\alpha)$; location parameter $=0$.

$$
\begin{gathered}
R\left(t_{m}\right) \approx 1-\operatorname{gauf}\left(\frac{t_{m} / \gamma-\alpha}{\sqrt{\alpha}}\right)+\left(\left[\frac{\left(t_{m} / \gamma-\alpha\right)^{2}}{\alpha}-1\right] /\right. \\
3 \sqrt{\alpha}) \exp \left[-\frac{1}{2} \frac{\left(t_{m} / \gamma-\alpha\right)^{2}}{\alpha}\right] / \sqrt{2 \pi}
\end{gathered}
$$

the Edgeworth expansion in terms of $s$-normal Cdf is used.

The components 1-5 have life distributions:

1. Weibull: $\hat{\theta}=400, \hat{B}=2, n=30$

2. Gamma: $\hat{\gamma}=100, \hat{\alpha}=3, n=100$

3, 4. Logistic: $\hat{\mu}=125, \hat{\sigma}=20, n=100$. The 10 smallest and 20 largest lives are censored.

5. Weibull: $\hat{\theta}=210, \hat{B}=3, n=100$

The 3-component networks all used only components of type one. They were chosen to see the effect of increasing system reliability.

\section{RESULTS AND MONTE CARLO SIMULATION}

Monte Carlo lower s-confidence limits and central $s$-confidence intervals are derived as follows:

The system reliability realizations obtained for each of the above three methods are inserted in the equation for system reliability for each system to obtain a system reliability realization. The process is repeated $N$ times. The system reliabilities are arranged in order of increasing magnitude. The ordered reliabilities partition the interval $[0,1]$ into $N+1$ equally probable intervals. We used $N+$ $1=1000$ for simplicity. From the ordered simulated reliabilities we can obtain any desired central $s$-confidence intervals or lower $s$-confidence limits, since the order statistics partition the range of reliability into $N+1$ equally probable intervals. To increase accuracy, the three Monte Carlo simulations were performed using $N+1=$ 1000 and the $s$-confidence limits obtained averaged. However this was not done for systems 4 and 5 for the double Monte Carlo method because of computer-time limitations.

Tables 1 and 2 compare central $s$-confidence intervals for $50 \%$ to $99 \% s$-confidence levels. The lower limit of a $\mathbf{9 9 \%} s$-confidence interval is also a $\mathbf{9 9 . 5 \%} 1$-tailed lower $s$-confidence limit and similarly for the other lower $s$-confidence limits. Due to computational difficulties for the double Monte Carlo method the 5-component case was rerun for all three methods with components 2 and 5 following the same Weibull failure model (calling the modification: system 6) with the results presented in Table 2. From Tables 1 and 2, the results of the two asymptotic methods tend to bracket those of the double Monte Carlo method. In general the bivariate method is conservative while the univariate asymptotic method is optimistic. To shed light on the Monte Carlo variability, Table $3 \mathrm{com}$ pares the ML point estimate of system reliability $\hat{R}_{s}\left(t_{m}\right)$ with median values of the system reliability realizations.

To measure the accuracy of the asymptotic methods each method is repeated many times with simulated test data from a component with known reliability. A single repetition is called a run. The percentage of times the $s$-confidence intervals covered the true system reliability is compared with the desired $s$-confidence level in Table 4 . For both methods all components were modeled by the Weibull distribution with sample size for simulated component test data $n=20$ for the univariate method and average sample size of 20 for the three components for the bivariate method. Table 4 indicates the bivariate method is conservative and in addition more accurate than the univariate method and much less sensitive to degradation due to high system reliability.

Table 5 compares CPU time for the three methods on the CDC 6600. Lannon [14] wrote computer programs that computed from sample data the parameter estimates, variance-covariance matrix, square root of variance

TABLE 1

\begin{tabular}{|c|c|c|c|c|c|c|c|c|c|c|c|c|c|c|c|c|c|c|}
\hline \multirow{3}{*}{$\frac{s \text {-Confidence }}{(\%)}$} & \multicolumn{6}{|c|}{3 Components in Series } & \multicolumn{6}{|c|}{$\begin{array}{l}1 \text { Component in Series } \\
\text { with } 2 \text { in Parallel }\end{array}$} & \multicolumn{6}{|c|}{3 Components in Parallel } \\
\hline & \multicolumn{3}{|c|}{ Lower Limits } & \multicolumn{3}{|c|}{ Upper Limits } & \multicolumn{3}{|c|}{ Lower Limits } & \multicolumn{3}{|c|}{ Upper Limits } & \multicolumn{3}{|c|}{ Lower Limits } & \multicolumn{3}{|c|}{ Upper Limits } \\
\hline & Biv & Dou & Uni & Biv & Dou & Uni & Biv & Dou & Uni & Biv & Dou & Uni & Biv & Dou & Uni & Biv & Dou & Uni \\
\hline 99 & .651 & .692 & .720 & .913 & .926 & .947 & .808 & .822 & .862 & .982 & .990 & 1.000 & .9981 & .9988 & .9990 & 1.0000 & 1.0000 & 1.0000 \\
\hline 95 & .697 & .730 & .741 & .898 & .908 & .914 & .847 & .865 & .880 & .973 & .981 & .992 & .9989 & .9992 & .9993 & 1.0000 & 1.0000 & 1.0000 \\
\hline 90 & .731 & .750 & .756 & .885 & .898 & .902 & .863 & .879 & .889 & .969 & .976 & .985 & .9992 & .9994 & .9994 & 1.0000 & 1.0000 & 1.0000 \\
\hline 80 & .751 & .772 & .773 & .871 & .885 & .887 & .883 & .896 & .900 & .964 & .971 & .971 & .9994 & .9995 & .9995 & .9999 & 1.0000 & 1.0000 \\
\hline 70 & .763 & .785 & .785 & .860 & .876 & .877 & .894 & .906 & .907 & .960 & .966 & .965 & .9995 & .9996 & 9996 & 9999 & 9999 & 1.0000 \\
\hline 60 & .774 & .794 & .793 & .853 & .867 & .868 & .903 & .915 & .912 & .955 & .961 & .960 & .9996 & .9997 & .9996 & .9999 & 9999 & 9999 \\
\hline 50 & .783 & .803 & .800 & .844 & .861 & .860 & .910 & .921 & .916 & .951 & .958 & .956 & .9996 & .9997 & .9997 & 9999 & 9999 & .9999 \\
\hline
\end{tabular}

Upper and Lower $s$-Confidence Limits for Reliability of 3-Component Systems 
TABLE 2

Upper and Lower $s$-Confidence Limits for Reliability of 5- and 25-Component Systems

\begin{tabular}{|c|c|c|c|c|c|c|c|c|c|c|c|c|c|c|}
\hline \multirow{3}{*}{$\frac{s \text {-Confidence }}{(\%)}$} & \multicolumn{4}{|c|}{ 5-Component System } & \multicolumn{4}{|c|}{ 25-Component System } & \multicolumn{6}{|c|}{ Modified 5-Component System } \\
\hline & \multicolumn{2}{|c|}{ Lower Limits } & \multicolumn{2}{|c|}{ Upper Limits } & \multicolumn{2}{|c|}{ Lower Limits } & \multicolumn{2}{|c|}{ Upper Limits } & \multicolumn{3}{|c|}{ Lower Limits } & \multicolumn{3}{|c|}{ Upper Limits } \\
\hline & Biv & Uni & Biv & Uni & Biv & Uni & Biv & Uni & Biv & Dou & Uni & Biv & Dou & Uni \\
\hline 99 & .811 & .859 & .977 & 1.000 & .805 & .847 & .967 & .995 & .811 & .845 & .859 & .974 & .980 & 1.000 \\
\hline 98 & .832 & .865 & .974 & .998 & .820 & .854 & .965 & .984 & .837 & .851 & .865 & .972 & .977 & .998 \\
\hline 97 & .840 & .869 & .972 & .991 & .828 & .858 & .963 & .982 & .842 & .855 & .869 & .970 & .975 & .991 \\
\hline 96 & .846 & .872 & .971 & .987 & .832 & .860 & .961 & .978 & .849 & .860 & .873 & .969 & .974 & .987 \\
\hline 95 & .849 & .874 & .970 & .984 & .842 & .862 & .960 & .977 & .852 & .862 & .874 & .968 & .973 & .984 \\
\hline 90 & .866 & .882 & .966 & .975 & .856 & .871 & .956 & .966 & .867 & .875 & .882 & .964 & .968 & .975 \\
\hline 80 & .885 & .893 & .960 & .965 & .873 & .882 & .950 & .956 & .883 & .888 & .893 & .958 & .962 & .965 \\
\hline 70 & .896 & .901 & .956 & .957 & .884 & .890 & .946 & .949 & .895 & .896 & .901 & .955 & .958 & .958 \\
\hline 60 & .902 & .906 & .952 & .952 & .892 & .895 & .942 & .943 & .903 & .903 & .906 & .951 & .954 & .952 \\
\hline 50 & .910 & .911 & .949 & .948 & .898 & .900 & .938 & .939 & .909 & .910 & .911 & .948 & .950 & .948 \\
\hline
\end{tabular}

TABLE 3

Median Value of $\hat{R}_{s}\left(t_{m}\right)$ Compared with ML Estimate of System Reliability $\hat{R}_{s}\left(t_{m}\right)$

\begin{tabular}{llllll}
\hline \hline & & & \multicolumn{3}{c}{ Method } \\
System & \multicolumn{1}{c}{ System Type } & $\hat{R}_{s}\left(t_{m}\right)$ & $\hat{R}_{s}\left(t_{m}\right)$ & $\begin{array}{c}\text { DOU } \\
\hat{R}_{s}\left(t_{m}\right)\end{array}$ & \multicolumn{1}{c}{$\hat{R}_{s}\left(t_{m}\right)$} \\
& & & & & \\
\hline 1 & 3 in series & .829 & .815 & .832 & .830 \\
2 & parallel series & .936 & .931 & .939 & .936 \\
3 & 3 in parallel & .9998 & .9998 & .9998 & .9998 \\
4 & 5 component & .929 & .931 & - & .929 \\
5 & 25 components & .919 & .921 & - & .920 \\
6 & 5 components (modified) & .929 & .931 & .932 & .930 \\
\hline
\end{tabular}

TABLE 4

True $s$-Confidence Levels

\begin{tabular}{|c|c|c|c|c|}
\hline & $\begin{array}{c}3 \text { in } \\
\text { Series } \\
\end{array}$ & $\begin{array}{c}\text { Parallel } \\
\text { Series }\end{array}$ & $\begin{array}{c}3 \text { in } \\
\text { Parallel }\end{array}$ & $\begin{array}{c}\text { Parallel } \\
\text { Series }\end{array}$ \\
\hline $\begin{array}{l}\text { System } \\
\text { Rel. }\end{array}$ & .684 & $\begin{array}{c}.843 \\
\text { (600 Runs) }\end{array}$ & .998 & $\begin{array}{c}.963 \\
\text { (700 Runs) }\end{array}$ \\
\hline \multicolumn{5}{|l|}{$\begin{array}{c}\text { Input } \\
s \text {-Conf. }\end{array}$} \\
\hline Level $(\%)$ & UNI [23] & UNI & UNI & BIV [14] \\
\hline 99 & 96.2 & 93.8 & 90.2 & 99.6 \\
\hline 90 & 86.2 & 80.8 & 70.5 & 92.6 \\
\hline 80 & 78.0 & 72.0 & 57.3 & 82.6 \\
\hline 70 & 68.2 & 64.7 & 46.2 & 73.0 \\
\hline 60 & 57.0 & 54.3 & 36.5 & 60.4 \\
\hline 50 & 48.3 & 47.0 & 27.7 & 49.0 \\
\hline
\end{tabular}

TABLE 5

Comparison of CPU Times on the CDC 6600 for the Three Methods (1000 Monte Carlo Samples) (25-Component System)

\begin{tabular}{ccc}
\hline \hline Method & Monte Carlo Sampling Only & Complete Job* \\
\hline BIV & $22 \mathrm{sec}$. & $50 \mathrm{sec}$. \\
DOU & $22 \mathrm{~min}$. & $23 \mathrm{~min}$. \\
UNI & $11 \mathrm{sec}$. & $40 \mathrm{sec}$.
\end{tabular}

*Including estimation of parameters and computation of variance covariance matrices (rough estimate of time). matrix, and resulting $s$-confidence intervals using the CDC 6600 for the bivariate asymptotic method when the failures were modeled by a 2-parameter Weibull. The total computation time was $22.3 \mathrm{sec}$ for 3 components and $29.5 \mathrm{sec}$ for an 8-component system using a Monte Carlo sample size of 1000 . Extrapolating from these results we obtained the values for the complete job for the 25 -component system.

The bivariate method is fast and accurate if the component failures are modeled by 2-parameter distributions with both parameters unknown. This method can use the exact distributions of parameter estimates when they are known [15]. For very sparse sample data, the double Monte Carlo method can be used, and would be much faster than indicated by Table 5 in this case.

\section{APPENDIX}

The variances of $\hat{R}\left(t_{m}\right)$ were calculated by using (2). For component \#1-

$$
\begin{aligned}
\operatorname{Var}\{ & \left\{\hat{R}_{1}\left(t_{m}, \hat{\theta}, \hat{B}\right)\right\}=D_{1}^{2} \operatorname{Var}\{\hat{B}\}+E_{1}^{2} \operatorname{Var}\{\hat{\theta}\} \\
& +2 D_{1} E_{1} \operatorname{Cov}\{\hat{\theta}, \hat{B}\} \\
D_{1} \equiv & -\left(t_{m} / \hat{\theta}\right)^{\hat{B}} \ln \left(t_{m} / \hat{\theta}\right) \hat{R}\left(t_{m}\right) \\
E_{1} \equiv & (\hat{B} / \hat{\theta})\left(t_{m} / \hat{\theta}\right)^{\hat{B}} \hat{R}\left(t_{m}\right) .
\end{aligned}
$$

Substituting the mission time, parameter estimates, and asymptotic variances and covariances from Harter \& Moore [11], we obtain $\sigma_{R_{1}}=.02877$. Similarly for component \#5 we obtain $\sigma_{\hat{R}_{5}}=.02224$.

For the two i.i.d. logistic components-

$$
\begin{aligned}
& \operatorname{Var}\left\{\hat{R}_{4}\left(t_{m}, \hat{\mu}, \hat{\sigma}\right)\right\}=D_{2}^{2} \operatorname{Var}\{\hat{\mu}\}+E_{2}^{2} \operatorname{Var}\{\hat{\sigma}\} \\
& \quad+2 D_{2} E_{2} \operatorname{Cov}\{\hat{\mu}, \hat{\sigma}\} \\
& D_{2} \equiv\left[\hat{R}\left(t_{m}\right)\right]^{-2}\left[\left(1-\hat{R}\left(t_{m}\right)\right)^{-1}-1\right] \pi /(\sqrt{3 \hat{\sigma}})
\end{aligned}
$$

$E_{2} \equiv\left[\hat{R}\left(t_{m}\right)\right]^{-2}\left[\left(1-\hat{R}\left(t_{m}\right)\right)^{-1}-1\right]\left[\pi\left(t_{m}-\hat{\mu}\right) /\left({\sqrt{3 \hat{\sigma}^{2}}}^{2}\right]\right.$. 
Substituting the parameter values, mission time $t_{m}$ and variances and covariances from Harter \& Moore [12], we obtain $\sigma_{\hat{R}_{4}}=\sigma_{\hat{R}_{3}}=.01929$.

For the component with a gamma failure distribution, we use the Edgeworth expansion of the Cdf in terms of the $s$-normal Cdf to obtain-

$$
\begin{aligned}
\operatorname{Var} & \left\{\hat{R}_{2}\right\} \approx \frac{\phi^{2}(\hat{\beta})}{n}\left[2 t_{m}^{2} /\left(\hat{\gamma}^{2} \sqrt{\hat{\alpha}}\right)\right. \\
& -(2 \hat{\alpha}-1)(1-\hat{\beta} /(2 \sqrt{\hat{\alpha}}))(1+3 \hat{\beta} /(2 \sqrt{\hat{\alpha}}))]
\end{aligned}
$$

where $\hat{\beta}=\left(t_{m} / \hat{\gamma}-\hat{\alpha}\right)$. Substituting the mission time and parameter estimates, we obtain $\sigma_{\hat{R}_{2}}=.01638$.

\section{REFERENCES}

[1] O.A. Bernhoff, "Confidence limits for system reliability based on component test data", MS thesis (GRE/MA/63-1), Air Force Institute of Technology; Wright-Patterson AFB, OH 45433 USA. 1963, AD-425845. Available from National Technical Information Service (NTIS); Department of Commerce, Springfield, VA 22161 USA.

[2] K.P. Berkbigler, J.K. Byers, "Systems reliability: Exact Bayesian intervals compared with fiducial intervals", IEEE Trans. Reliability, vol R-24, 1975 Aug, pp 199-200.

[3] J.E. Bilikam, A.H. Moore, "Estimation of reliability from multiple independent grouped censored samples with failure times known”, IEEE Trans. Reliability, vol R-27, 1978 Dec, pp 329-331.

[4] J.E. Bilikam, A.H. Moore, "Estimation of mission reliability from multiple independent grouped censored samples", IEEE Trans. Reliability, vol R-26, 1977 Apr, pp 57-60.

[5] R.J. Buehler, "Confidence intervals for the product of two binomial parameters", American Statistical Assoc., vol 52, 1957 Dec, pp 482-493.

[6] B. Efron, "Bootstrap method: another look at the jackknife", Annals of Statistics, vol 7, 1979 Jan, pp 1-26.

[7] T.R. Gatliffe, "Accuracy analysis for a lower confidence limit procedure for system reliability", Naval Postgraduate School, Monterey, California, 1976, AD-A031817. Available from NTIS (see [1]).

[8] H.L. Harter, A.H. Moore, "Maximum-likelihood estimation of the parameters of gamma and Weibull populations from complete and censored samples", Technometrics, vol 7, 1965 Nov, pp 639-643.

[9] H.L. Harter, A.H. Moore, "Local-maximum likelihood estimation of the parameters of three parameter lognormal populations from complete and censored samples", American Statistical Assoc., vol 61, 1966 Sep, pp 842-851.

[10] H.L. Harter, A.H. Moore, "Iterative maximum-likelihood estimation of the parameters of normal populations from singly and doubly censored samples", Biometrika, vol 53, 1966 Aug, pp 205-213.

[11] H.L. Harter, A.H. Moore, "Asymptotic variances and covariances of maximum-likelihood estimators, from censored samples, of the parameters of Weibull and gamma populations", Annals Mathematical Statistics, vol 38, 1967 Apr, pp 557-570.

[12] H.L. Harter, A.H. Moore, "Maximum-likelihood estimation from censored samples, of the parameters of a logistic distribu'tion", American Statistical Assoc., vol 62, 1967 Jun, pp 675-682.

[13] H.L. Harter, A.H. Moore, "Maximum-likelihood estimation from doubly censored samples, of the parameters of the first asymptotic distribution of extreme values", American Statistical Assoc., vol 63, 1968 Sep, pp 889-901.
[14] R.G. Lannon, "A Monte Carlo technique for approximating system reliability confidence limits using the Weibull distribution", MS thesis (GAM/MA/72-2), AFIT, WrightPatterson AFB, Ohio, 1972, AD-743633. Available from NTIS (see [1]).

[15] L.L. Levy, A.H. Moore, "A Monte Carlo technique for obtaining system reliability confidence limits from component test data, IEEE Trans. Reliability, vol R-16, 1967 Sep, pp 69-72.

[16] M.O. Locks, "Monte Carlo Bayesian system reliability-and MTBF-confidence assessment", Air Force Flight Dynamics Laboratory, Wright-Patterson AFB, Ohio, AFFDL-TR-75-144, AD-A057068. Available from NTIS (see [1]).

[17] M.O. Locks, "Monte Carlo Bayesian system reliability-and MTBF-confidence assessment II", vol I: Theory, vol II: SPARCS-2 Users Manual, Air Force Flight Dynamics Laboratory, Wright-Patterson AFB, Ohio, AFFDL-TR-78-18, AD-A025820. Available from NTIS (see [1]).

[18] S.C. Lutton, "A Monte Carlo technique for approximating system reliability confidence limits from component failure test data", MS thesis (GRE/MA/67-9). Available from Air Force Institute of Technology (AFIT); Wright-Patterson AFB, Ohio 45433 USA.

[19] A. Madansky, "Approximate confidence limits for the reliability of series and parallel systems", Technometrics, vol 7, 1965 Nov, pp 495-506.

[20] N.R. Mann, F.E. Grubbs, "Approximately optimum confidence bounds for system reliability based on component test data", Technometrics, vol 16, 1974 Aug, pp 335-347.

[21] A.H. Moore, "Extension of Monte Carlo technique for obtaining system reliability confidence limits from component test data", Proc. National Aerospace Electronics Conf, 1965 May, pp 459-463.

[22] T.S. O'Neil, "System reliability assessment from its components", Applied Statistician, vol 21, 1972, pp 297-320.

[23] R.B. Putz, "A Univariate Monte Carlo technique to approximate reliability confidence limits of systems with components characterized by the Weibull distribution", MS thesis (GOR/MA/79D-7), AFIT. Available from NTIS (see [1]).

[24] M.D. Springer, W.E. Thompson, "Bayesian confidence limits for the product of $N$ binomial parameters", Biometrika, vol 53, 1966 Dec, pp 611-613.

[25] M.D. Springer, W.E. Thompson, "Bayesian confidence limits for the reliability of cascade exponential systems, IEEE Trans. Reliability, vol R-16, 1967 Sep, pp 86-89.

[26] M.D. Springer, W.E. Thompson, "Bayesian confidence limits for reliability of redundant systems when tests terminated at first failure", Technometrics, vol 10, 1968 Feb, pp 29-36.

[27] Wash-1400 "Reactor Safety Study-Appendix 2-Fault Trees", 1975 Oct, pp 575. PB-248 203/2GI. Available from NTIS (see [1]).

\section{AUTHORS}

Albert H. Moore; Air Force Institute of Technology (AFIT/ENC), Wright-Patterson AFB OH 45433 USA.

Albert H. Moore: For biography see vol R-22, 1973 Dec, p 264. He was promoted to the rank of Professor of Mathematics at the U.S. Air Force Institute of Technology, Wright-Patterson AFB in 1974.

H. Leon Harter; Department of Mathematics, Wright State University, Dayton OH 45435 USA.

H. Leon Harter: For biography see vol R-22, $1973 \mathrm{Dec}, \mathrm{p} 264$. Following the disestablishment of ARL in 1975, he transferred to the Air Force Flight Dynamics Laboratory until retirement in 1978. Since 1979 he has been a Research Professor of Mathematics at Wright State University. In 1977 he was elected a member of the International Statistical Institute. 
Robert C. Snead; Tracor, Inc., 6400 Tracor Lane, Austin TX 78721 USA.

Robert C. Snead was born in Albany GA on 1949 Jan 16. He received his BS in economics from the USAF Academy in 1970 and the MS degree in Operations Research from the Air Force Institute of Technology in 1978. In 1970, he joined the U.S. Air Force and duty included program- mer and systems analyst. Since 1978 he has been a financial analyst with Tracor, Inc.

Manuscript TR79-30 received 1979 February 20; revised 1980 February 13.

\section{Book Review}

\section{Software Tools}

Brian W. Kernighan and P. J. Plauger, 1976, \$10.95 paperback, $338 \mathrm{pp}$.

Addison-Wesley Publishing Company; Reading, Massachusetts USA.

ISBN: 0-201-03669-X.

\section{Table of Contents}

1. Getting Started
2. Filters
3. Files
4. Sorting
5. Text Patterns
6. Editing
7. Formatting
8. Macro Processing
9. A Ratfor-Fortran Translator
Epilogue
Appendix: Primitives and Symbolic Constants
Indexes

$28 \mathrm{pp}$

$22 \mathrm{pp}$

$38 \mathrm{pP}$

$30 \mathrm{pp}$

$18 \mathrm{pp}$

$56 \mathrm{pp}$

$32 \mathrm{Pp}$

$34 \mathrm{PP}$

$34 \mathrm{pp}$

$4 \mathrm{PP}$

$2 \mathrm{pp}$

$13 \mathrm{pp}$

This is a good book. It teaches good programming by example rather than by listing a set of cookbook rules which must be followed. The more you study it, the better it becomes. It is not a textbook, although it could be used to supplement the teacher's role in a course on programming. In order to use the book effectively, one needs some experience in programming.

Virtually all of the material is related to text processing, rather than, say, to compilers or mathematical problems. Some of this comes about because of the authors close relation to "the C programming language" (see the review, in this issue, of the book by that name) which has but few I/O facilities. The authors explain: "It might appear from this outline that we stress text manipulation too heavily. Yet computing is not all number-crunching, nor is it the 'compilers, assemblers and loaders' so hastily treated in many second courses in programming. A large part of what programmers do every day is text processing - editing program source, preparing input data, scanning output, writing documentation. ..." Some of the good points made by the authors (sometimes implicitly) are: 1) A well written program is its own documentation. And conversely, a program which requires extensive documentation probably could be rewritten to be much clearer - without the extensive documentation. 2) Good programming style, rather than blind application of particular rules or techniques, is what produces good programs. Bad programs can be written which follow all the rules. 3) Don't second-guess the computer, or yourself, on how to create efficient code. Don't outsmart yourself trying to create efficient code. First, write good understandable code. Then, let the computer tell you where it is spending most of its time - and thus where moreefficient code will help.

Any programmer who is not familiar with another of the authors' books: The Elements of Programming Style (MCGraw Hill, 1974) ought to buy it and read it. It is inexpensive (approx \$3), short, and easy to read. It was reviewed in vol R-23, 1974 Dec, pp 342-343.

Even though the examples come largely from text processing, the principles apply widely. The book is not expensive, and you can learn alot from it. You can apply the principles and style the day you begin reading the book. There is no need to turn the programming department upside down with a complete reorganization. $*^{*} *$

\section{The C Programming Language}

Brian W. Kernighan and Dennis M. Ritchie, 1978, $\$ 10.95$ paperback, $228 \mathrm{pp}$.

Prentice-Hall, Inc.; Englewood Cliffs, NJ 07632 USA. ISBN: 0-13-110163-3; LCCCN: 77-28983.

\section{Table of Contents}

1. A Tutorial Introduction

2. Types, Operators, and Expressions

3. Control Flow

4. Functions and Program Structure

5. Pointers and Arrays

6. Structures

7. Input and Output

8. The UNIX System Interface

Appendix A. C Reference Manual Index
$27 \mathrm{PP}$

$18 \mathrm{pp}$

$14 \mathrm{pp}$

$24 \mathrm{PP}$

$30 \mathrm{pp}$

$24 \mathrm{pp}$

$16 \mathrm{pp}$

$20 \mathrm{pp}$

$42 \mathrm{pp}$

$7 \mathrm{PP}$
New small programming languages are being touted all over the land. Pascal is receiving a big push, perhaps because of its suitability for microcomputers which are likewise becoming ubiquitous. This is THE book on the C language. Appendix A defines the language. " $\mathrm{C}$ " has not been used widely enough to become endowed with all the variants which wide use creates.

Some claim that " $\mathrm{C}$ " is much better than Pascal because it allows the programmer more flexibility and introduces some shorthand notation for widely used, cumbersome operations (shades of APL). A favorable review of "C", by Tim Chase, appeared in: Information System News, Monday 1980 May 19, p 24. Chase states that " $C$ " can now be implemented on IBM/370, DEC $\mathrm{PDP} / 11$, and the $\mathrm{HP} / 1000$.

Undoubtedly " $\mathrm{C}$ " will be a useful language for creating high quality programs. Just as undoubtedly, it can be used to create very bad programs. There is a running argument (for reliable software) over how much flexibility a programmer should be allowed by the programming language, and how compact the language should be. APL is probably too compact. Whether the special constructs in " $\mathrm{C}$ " will be a bane or boone, only continued usage will tell. The $C$ language does have many advantages; it does have some disadvantages (e.g. you have to create most of your own $\mathrm{I} / \mathrm{O}$ routines, and it needs extending).

If you want to learn about " $C$ " from the horse's mouth, this is the book to get. But beware about hoping that the programming language you use will solve all quality problems in programs. It won't. 